\title{
A SILICON PHOTODIODE BASED GAS PROPORTIONAL SCINTILIATION COUNTER
}

\section{A. J. de Campos}

Departamento de Física, Universidade de Coimbra, 3000 Coimbra, Portugal

\section{Abstract}

A commercial available Schottky barrier silicon photodiode is coupled to a Gas Proportional Scintillation counter through a quartz window and is used as an alpha particle room temperature detector. An Ar+N2(2\%) gas mixture at 1470 Torr is used. Resolutions of $3.2 \%$ are achieved. A discussion of the results obtained is presented and future improvements considered.

\section{Introduction}

During the last years efforts have been made in order to replace the cumbersome conventional photomultiplier used in scintillation detectors with smaller light sensors - photodiodes and photoionization detectors.

Standard scintillators ( $N a I$ and CsI) have been coupled to surface barrier [1], Si(Li) [2] and silicon avalanche [3] photodiodes. Gas scintillation and gas proportional scintillation counters have been coupled to vacuum photodiodes [4] and to photoionization detectors $[5,6,7]$ with special success in the case of proportional scintillators due to the large light intensity.

Silicon photodiodes can in principle be used as well with gas proportional scintillation counters
(GPSC). In spite of its appealing simplicity systems of this type have not yet been tried. Having in mind the development of silicon photodiode based portable GPSC's we carried out the present work.

\section{Experimental set-up}

A standard uniform field gas proportional scintillation counter is shown in Fig. 1 with two grids $6 \mathrm{~mm}$ apart. The grids were made of AISI-304 stainless steel with a mesh of 100 c.p.cm2. The scintillation light passes through a quartz ( Spectrosil-A) window and is detected with a commercial available ( United Detector Technology, California) Schottky barrier PIN silicon photodiode, model PIN-25, with a sensitive area of $6 \mathrm{~cm} 2$. Both the photodiode and the preamplifier ( Hewlett-Packard model HP 5554-A ) are operated at room temperature.

Since Si-photodiodes spectral response falls to zero below $200 \mathrm{~nm}$, even for UV types, they cannot be used with GPSC's using pure noble gas fillings. However it has been shown [8] that Ar-N2 mixtures emit light above that $200 \mathrm{~nm}$ threshold. Thus we have filled the detector with an $\mathrm{Ar}+\mathrm{N} 2(2 \%)$ gas mixture; the gas was kept at 1470 Torr and $17^{\circ} \mathrm{C}$.

The GPSC was excited with Th-C alpha particles dissipating $8.5 \mathrm{MeV}$ in the gas.

The electronics diagram is shown in Fig. 2 with the experimentally values set for minimum SNR. Both the

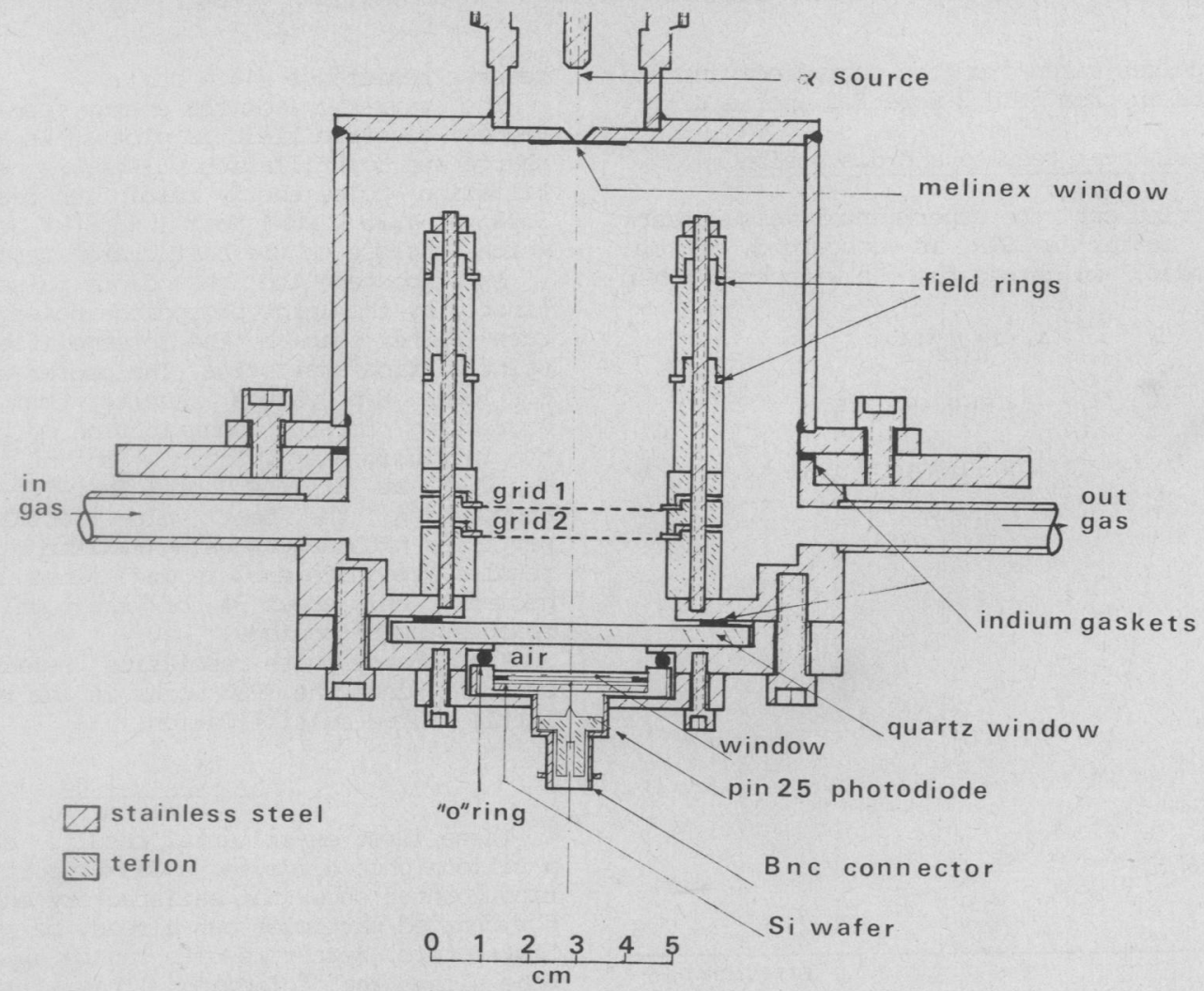

Fig.1 GPSC WITH COUPLED PHOTODIODE 


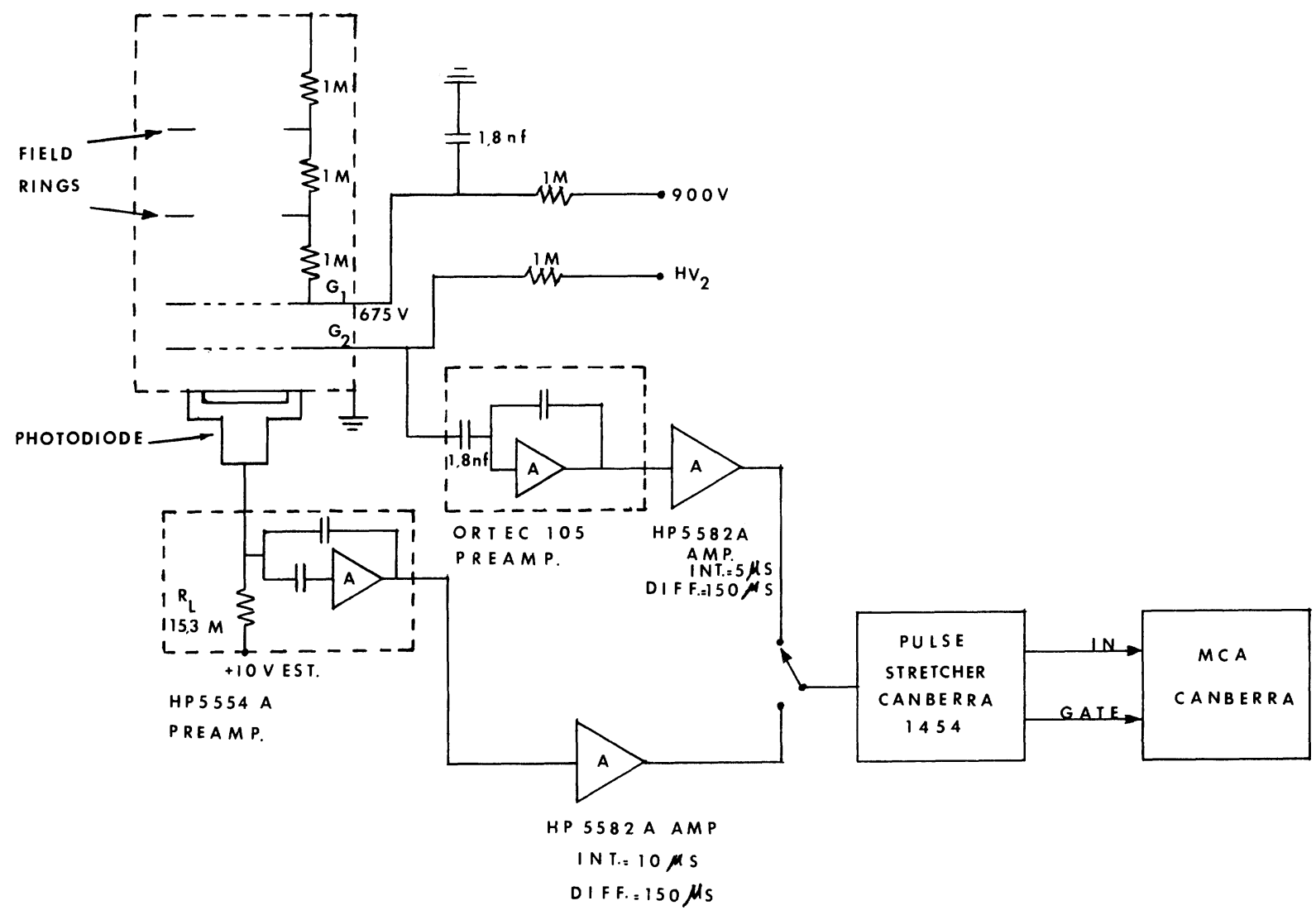

FIG.2 ELECTRONICS DIAGRAM WITH SNR OPTIMIZED VALUES

scintillation pulses taken from the photodiode and the charge pulses taken from grid 2 were fed into a MCA.

\section{Experimental results and discussion}

We have carried out the experimental measurements with the best, as far as SNR is concerned, of two PIN-25 photodiodes, which was the one with the higher

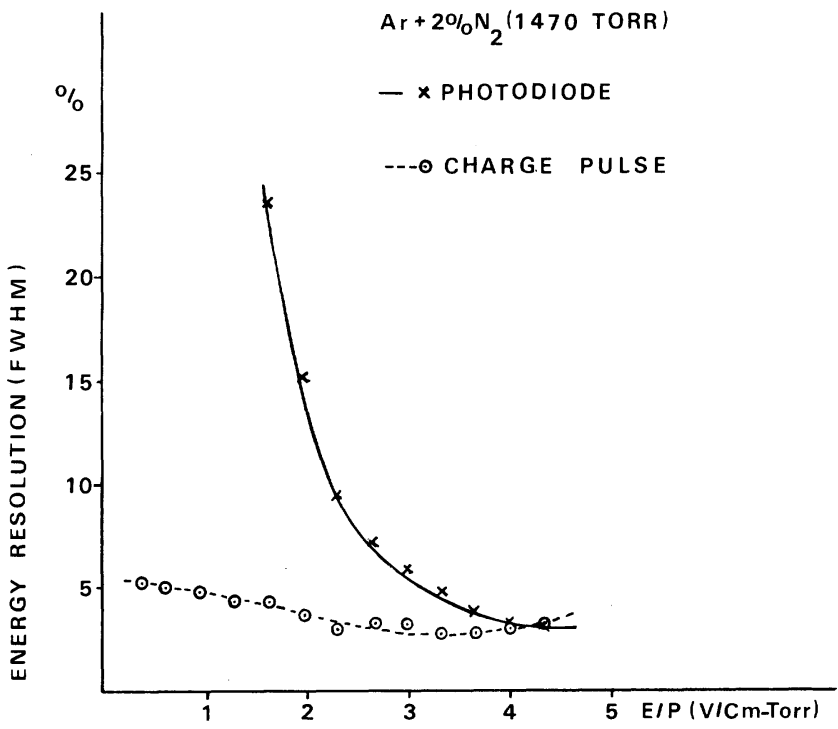

FIg.3 ENERGY RESOLUTION reverse resistance (18 $\mathrm{M} \mathrm{ohm})$.

The variation of the energy resolution with the reduced electric field is plotted in Fig. 3 for both charge and scintillation pulses. As shown, the scintillation pulse energy resolution reaches a figure of $3.2 \%$ at $4.35 \mathrm{~V} \mathrm{~cm}-1$ Torr -1 (3.8 kV between the grids) which is close to the best charge resolution.

As expected the photodiode pulse resolution is limited by the large photodiode noise at room temperature rather than by the preamplifier noise or the scintillation statistics. The poorer energy resolution for lower $E / p$ values results from the smaller SNR. Correcting for the contribution of the system noise the intrinsic energy resolution of the counter would be $2.1 \%$ at $\mathrm{E} / \mathrm{p}=4 \mathrm{~V} \mathrm{~cm}-1$ Torr -1 . This value is approaching the best value obtained for alpha particles with GPSC's using photomultipliers [9]. These results are quite encouraging since under the present geometry only about 5\% of the total secondary light reaches the photodiode.

The charge pulse resolution remains approximately constant since the GPSC works in the region of none or little charge multiplication.

\section{Conclusions}

These first experimental results obtained coupling a silicon photodiode to a Gas Proportional Scintillation counter show that satisfactory energy resolutions for charged particles can already be obtained at room temperature. Better results can be expected if work is done along the following lines: improvement in the optical solid angle, the photodiode spectral response and the noise of the system. 


\section{Aknowledgements}

We are indebted to Prof. C. A. N. Conde for his interest and useful suggestions and discussions and to Mr. C. J. N. Gomes for the drawings. Financial support from Instituto Nacional de Investigação Cientifica is acknowledged. Our thanks are also due to the Deutsche Gesellschaft für Technische Zusammenarbeit ( GTZ ) for the donation of some equipment we used.

\section{References}

[1] N. G. Blamires, "Combination of a scintillator and a semiconductor photodiode for nuclear particle detection", Nucl. Instr. and Meth. , $24,441-444$, (1963).

[2] J. E. Bateman and F. E. Özsan, "A cryogenic scintillator-photodiode detector for penetrating charged particles", Nucl. Instr. and Meth., 108 , 403-407, (1973).

[3] G. Entine, G. Reiff, M. Squillante, H. B. Serreze, S. Lis and Gerald Huth, "Scintillation detectors using large area silicon avalanche photodiodes", IEEE Trans. Nucl. Sci., NS-30, 431-435, (1983).

[4] J. C. van Staden, J. Foh, M. Mutterer, J. Pannicke, K. P. Schelhaas and J. P. Theobald, "A vacuum photodiode as light sensing element for gas scintillation counters", Nucl. Instr. and Meth., 157, 301-304, (1978).

[5] A. J. P. L. Policarpo, "Coupling the gas scintillation proportional counter to photoionization detectors", Nucl. Instr. and Meth., 153 , 389-391, (1978).

[6] G. Charpak, A. Policarpo and F. Sauli, "The photoionization proportional scintillation chamber" , IEEE Trans. Nucl. Sci., NS-27, 212-215, (1980).

[7] M. Salete S. C. P. Leite , A. J. P. L. Policarpo , M. Alegria Feio and M. A. F. Alves, "The use of rare gas mixtures in the photoionization proportional scintillation chamber", Nucl. Instr. and Meth., 177, 609-611, (1980).

[8] A. J. P. L. Policarpo, M. A. F. Alves and C. A. N. Conde, "The argon-nitrogen proportional scintillation counter" , Nucl. Instr. and Meth., 55 , 105$-119,(1967)$.

[9] C. A. N. Conde, L. Requicha Ferreira and M. Fátima A. Ferreira, "The secondary scintillation output of Xenon in a uniform field gas proportional scintillation counter", IEEE Trans. Nucl. Sci. NS-24, 221-224, (1977). 